\title{
Authors' reply: Cognitive and emotional factors are involved in body-image distortion
}

\section{Guido K. Frank and Janet Treasure}

We appreciate the opportunity to discuss body image further than was possible in our original Primer (Anorexia nervosa. Nat. Rev. Dis. Primers 1, $15074(2015))^{1}$ in response to the correspondence by Prof. Dakanalis and colleagues (Body-image distortion in anorexia nervosa. Nat. Rev. Dis. Primers 2, $16026(2016))^{2}$.

Body-image distortion (BID) is a frequently referenced symptom in those with anorexia nervosa (AN) and is part of the diagnostic criteria for $\mathrm{AN}^{3}$. Its underpinnings, neurobiologically or psychologically, are still under investigation and debate is ongoing whether cognitive, emotional or perceptual factors primarily drive this "disturbance in the way in which one's body weight or shape is experienced" (REF. 3). Early research noted that attitudes towards one's body - such as intense loathing of the body - were extreme in individuals with AN; furthermore, thin-body messages in the media have been shown to influence how individuals with AN view their body ${ }^{4}$. Indeed, over the past two decades, researchers have investigated whether differences in sensory perception contribute to BID in AN.

One school of thought is that AN is associated with a multisensory impairment of body perception, as Dakanalis et al. ${ }^{2}$ pointed out and as reviewed by Gaudio et al. ${ }^{5}$. That review ${ }^{5}$ searched the literature for possible alterations in body schema and perception in eating disorders and included studies on tactile perception, interoception and body perception. The authors concluded that AN is associated with an altered capacity to process and integrate bodily signals. However, not all studies support the notion of altered sensory processing in AN and their review might have been too selective ${ }^{6,7}$. Other research went further and used functional MRI (fMRI) to identify brain regions that are involved in BID; many of those studies were reviewed by Gaudio and Quattrocchi ${ }^{8}$. Dakanalis et al. ${ }^{2}$ deduct from these and other studies that there is now good evidence that BID is related to alterations of the prefrontal cortex, the insula and the amygdala and that the 'perceptive' component of BID is related to alterations of the parietal lobes, implicating spatial and body representations, body ownership and other features that require multisensory integration and visuospatial processing.

However, increasing evidence points in the other direction. That is, perceptual disturbance might not drive BID; instead, a cognitive drive for thinness and fear of weight gain might be at play. Research that tested sensory and non-sensory components of BID has shown no differences in sensory sensitivity between individuals with an eating disorder and controls, but that cognitive-emotional factors such as selfideal discrepancy and body dissatisfaction drive body-size estimation ${ }^{9}$. One study used computer-based body-distortion techniques and did not find that patients with AN overestimated their body dimensions ${ }^{10}$. Another study in which individuals manipulated their body size on a computer screen found that individuals with AN did overestimate their body size and that this process was driven by desire for thinness and body dissatisfaction, independent of body mass index - making a perceptual distortion unlikely ${ }^{11}$. One report specifically suggested that cognitive dysfunction in individuals with AN during the ill state might drive $\mathrm{BID}^{12}$, which is supported by other studies that suggested that there is a psychotic-delusional component to the overvalued ideas of thinness, particular in restricting-type $\mathrm{AN}^{13}$.

An interesting concept that was developed in this context is that of thought-shape fusion ${ }^{14}-$ a cognitive distortion in which an individual experiences increasing body size after thinking about certain types of food. Thought-shape fusion suggests that a top-down mechanism is driving body perception. Situational cues such as eating, thinking of one's appearance and thinking about how one is perceived by others have also been found to drive body image. Other factors that influenced body-size estimation and drove preoccupation with dieting in individuals with AN include exposure to 'round' body shapes ${ }^{15}$.
Overall, general perceptual deficits in AN have not been uniformly identified, and whether perceptual deficits contribute to BID has not been conclusively determined. Although using fMRI to assess brain activation during body-image tasks might support the hypothesis of perceptual alterations in AN, the technique only measures blood flow. Accordingly, without good basic science or an animal model to help us to interpret the data, we are often left with conjecture - a general problem in brain imaging research in psychiatry. In summary, there remains uncertainty as to whether BID in AN is a primary perceptual problem or whether it is driven by cognitive-emotional processes; specifically, dissatisfaction with selfevaluation could manifest as a perfectionistic pursuit of thinness to attain and exceed a purportedly society-driven but unrealistic thin ideal. Problems with body image, which are an important source of distress in society, demand further research.

Guido K. Frank is at the Pediatric Mental Health Institute, Children's Hospital Colorado, Department of Psychiatry, University of Colorado Anschutz Medical Campus, Colorado, USA.

Janet Treasure is at King's College London, Department of Psychological Medicine, Institute of Psychiatry, Psychology and Neuroscience (IoPPN), London SE5 8AF, UK.

Correspondence to J.T. janet.treasure@kcl.ac.uk

Article number: 16027 doi: $10.1038 /$ nrdp. 2016.27 Published online 21 April 2016

Treasure, J. et al. Anorexia nervosa. Nat. Rev. Dis. Primers 1, 15074 (2015)

2. Dakanalis, A. et al. Body-image distortion in anorexia nervosa. Nat. Rev. Dis. Primers 2, 16026 (2016). American Psychiatric Association. Diagnostic and Statistical Manual of Mental Disorders, Fifth Edition (DSM-5) (American Psychiatric Publishing, 2013).

4. Hamilton, K. \& Waller, G. Media influences on body size estimation in anorexia and bulimia. An experimental study. Br. J. Psychiatry 162, 837-840 (1993).

5. Gaudio, S., Brooks, S. J. \& Riva, G. Nonvisual multisensory impairment of body perception in anorexia nervosa: a systematic review of neuropsychological studies. PLOS ONE 9, e110087 (2014).

6. Goldzak-Kunik, G., Friedman, R., Spitz, M., Sandler, L. \& Leshem, M. Intact sensory function in anorexia nervosa. Am. J. Clin. Nutr. 95, 272-282 (2012).

7. Waldman, A., Loomes, R., Mountford, V. A. $\Sigma$ Tchanturia, K. Attitudinal and perceptual factors in body image distortion: an exploratory study in patients with anorexia nervosa. J. Eat. Disord. 1, 17 (2013).

8. Gaudio, S. \& Quattrocchi, C. C. Neural basis of a multidimensional model of body image distortion in anorexia nervosa. Neurosci. Biobehav. Rev. 36 , 1839-1847 (2012).

9. Cash, T. \& Deagle, E. The nature and extent of bodyimage disturbances in anorexia nervosa and bulimia nervosa: a meta-analysis. Int. J. Eat. Disord. 22 , 107-125 (1997)

10. Hennighausen, K., Enkelmann, D., Wewetzer, C. \& Remschmidt, $\mathrm{H}$. Body image distortion in anorexia nervosa - is there really a perceptual deficit? Eur. Child Adolesc. Psychiatry 8, 200-206 (1999). 


\section{CORRESPONDENCE}

11. Hagman, J. et al. Body size overestimation and its association with body mass index, body dissatisfaction, and drive for thinness in anorexia nervosa. Eat. Weight Disord. 20, 449-455 (2015).

12. Epstein, J. et al. Neurocognitive evidence favors "top down" over "bottom up" mechanisms in the pathogenesis of body size distortions in anorexia nervosa. Eat. Weight Disord. 6, 140-147 (2001)

13. Konstantakopoulos, G. et al. Delusionality of body image beliefs in eating disorders. Psychiatry Res. 200 482-488 (2012)
14. Shafran, R. \& Robinson, P. Thought-shape fusion in eating disorders. Br. J. Clin. Psychol. 43, 399-408 (2004).

15. Cazzato, V. et al. The effects of body exposure on self-body image and esthetic appreciation in anorexia nervosa. Exp. Brain Res. 234, 695-709 (2016).

Competing interests

$\mathrm{J}$.T. has received royalties from authored books and an honorarium from Lilly for a presentation at the Diabetes Meeting 2015. She has received research grants from the
National Institute for Health Research (NIHR) under the Research for Patient Benefit (RfPB) programme, Mental Health Biomedical Research Centre at South London, the Maudsley NHS Foundation Trust, King's College London, the Swiss Anorexia Nervosa Foundation, Guy's and St Thomas' Charity and the Psychiatry Research Trust. G.K.F. has received funding from the National Institute of Mental Health, the Davis Foundation Award of the Klarman Family Foundation, the Obesity and Nutrition Research Center, University of Pittsburgh, Pennsylvania, USA, and has served as a consultant for Shire. 